Portland State University

PDXScholar

$11-8-2002$

\title{
Modified Bell-Plesset Effect with Compressibility: Application to Double-Shell Ignition Target Designs
}

\author{
Peter A. Amendt \\ Lawrence Livermore National Laboratory \\ J. D. Colvin \\ Lawrence Livermore National Laboratory \\ John D. Ramshaw \\ Portland State University, jdramshaw@yahoo.com \\ H. F. Robey \\ Lawrence Livermore National Laboratory \\ O. L. Landen \\ Lawrence Livermore National Laboratory
}

Follow this and additional works at: https://pdxscholar.library.pdx.edu/phy_fac

Part of the Physics Commons

Let us know how access to this document benefits you.

\section{Citation Details}

P. Amendt, J.D. Colvin, J.D. Ramshaw, H.F. Robey, and O.L. Landen, "Modified Bell-Plesset effect with compressibility: Application to double-shell ignition target designs," Phys. Plasmas 10, 820 (2003)

This Article is brought to you for free and open access. It has been accepted for inclusion in Physics Faculty Publications and Presentations by an authorized administrator of PDXScholar. Please contact us if we can make this document more accessible: pdxscholar@pdx.edu. 


\title{
Modified Bell-Plesset effect with compressibility: Application to double-shell ignition target designs
}

\author{
Peter Amendt, J. D. Colvin, J. D. Ramshaw, H. F. Robey, and O. L. Landen \\ Lawrence Livermore National Laboratory, Livermore, California 94551
}

(Received 29 August 2002; accepted 8 November 2002)

\begin{abstract}
The effect of spherical convergence on the fluid stability of collapsing and expanding bubbles was originally treated by Bell [Los Alamos Scientific Laboratory Report No. LA-1321 (1951)] and Plesset [J. Appl. Phys. 25, 96 (1954)]. The additional effect of fluid compressibility was also considered by Bell but was limited to the case of nonzero density on only one side of a fluid interface. A more general extension is developed which considers distinct time-dependent uniform densities on both sides of an interface in a spherically converging geometry. A modified form of the velocity potential is used that avoids an unphysical divergence at the origin [Goncharov et al., Phys. Plasmas 7, 5118 (2000); Lin et al., Phys. Fluids 14, 2925 (2002)]. Two consequences of this approach are that an instability proposed by Plesset for an expanding bubble in the limit of large interior density is now absent and application to inertial confinement fusion studies of stability becomes feasible. The model is applied to a proposed ignition double-shell target design [Amendt et al., Phys. Plasmas 9, 2221 (2002)] for the National Ignition Facility [Paisner et al., Laser Focus World 30, 75 (1994)] for studying the stability of the inner surface of an imploding high- $Z$ inner shell. Application of the Haan [Phys. Rev. A 39, 5812 (1989)] saturation criterion suggests that ignition is possible. (C) 2003 American Institute of Physics. [DOI: 10.1063/1.1543926]
\end{abstract}

\section{INTRODUCTION}

The growth of incompressible fluid perturbations in a spherically converging geometry is a well-known consequence of mass conservation. Bell, ${ }^{1}$ Plesset $^{2}{ }^{2}$ and Birkhoff ${ }^{3}$ independently described this effect in the early 1950s using a velocity potential treatment. Bell considered further the effect of compressibility on either side of a fluid interface. In an unpublished memorandum from Los Alamos Scientific Laboratory in 1982, Fisher attempted to generalize Bell's treatment to include the effect of nonzero densities with distinct rates of uniform compression on both sides of a fluid interface. ${ }^{4}$ Fisher's apparent goal was to apply the model to imploding shells in inertial confinement fusion (ICF) for studying stability.

An unfortunate feature of these previous treatments on the so-called "Bell-Plesset" effect is the choice of velocity potential adopted. In these studies a term with divergent behavior at the origin was included in the velocity potential which is tantamount to introducing a source or sink of mass at $r=0$ (see Sec. II). Unless the density of the material interior to the collapsing bubble or imploding shell is negligibly small misleading results can ensue. For example, the peak density of the imploded fuel in an ICF target can approach the peak shell density at minimum volume and invalidate the implicit assumption of a low density cavity. Collapse of a vapor-generated bubble in medical applications is another example where the interior density approaches the ambient fluid density. ${ }^{5}$ For these reasons a modification to the customary form of the velocity potential is preferred.

Aside from an interest in understanding the role of the Bell-Plesset effect in general, the techniques developed for studying collapsing and expanding bubbles have a poten- tially important application to studies of unstable interfaces in proposed ignition targets. A favored technique to date has been a two-dimensional simulation of an imploding capsule with an imposed single-mode small-amplitude perturbation. A suite of calculations is then performed over a range of modes to generate a linear growth-factor spectrum on a particular interface at a time of interest, e.g., instant of peak neutron production. This spectrum is convolved with an initial surface spectrum and summed in quadrature to estimate the annular extent of perturbation growth on an unstable interface. The growth of a group of modes may enter the nonlinear regime at which point a standard saturation model can be invoked to further describe the perturbation behavior. ${ }^{6}$

As clear-cut as this procedure may seem, implementation is often very challenging in practice, particularly for higher mode numbers or short wavelength perturbations. In two-dimensional radiation-hydrodyamic simulations control of mesh instabilities that can overwhelm or compromise the perturbation waveform of interest is paramount. To this end various numerical filters are invoked in which particular care is exercised to not appreciably affect the intrinsic growth of the perturbation. This procedure has been successfully applied to the case where perturbations that grow on the ablation surface and feedthrough to the inner surface are amplified upon deceleration onset. ${ }^{7}$ The ablation and feedthrough processes both act to strongly filter high mode number perturbations, thereby usually avoiding the numerical challenge of accurately describing the growth of a high-mode perturbation. In contrast to this feed through scenario, the case of intrinsic growth of perturbations initially residing on the inner surface is more challenging to capture numerically. In the absence of ablation stabilization - as well as an absence of 
density gradient stabilization if the interface is between two distinct materials-high mode-number perturbations have high linear growth-factors. Our experience in this regime has been that numerical filtering is necessary to preserve the form of the perturbation but at the expense of possibly compromising the integrity of the growth-factor result.

Given these constraints on reliably carrying through such calculations a complementary approach is to combine a perturbation analysis with detailed one-dimensional radiation-hydrodynamics simulations of the unperturbed shell behavior for extracting detailed information on the perturbation growth. This procedure is essential for understanding linear perturbation growth on the inner surface of the interior shell of a double-shell target where credible twodimensional single-mode growth-factor calculations have proven elusive to date.

In Sec. II we use a recently introduced form for the velocity potential ${ }^{8}$ and carry through the linear perturbation analysis. In Sec. III we generate a linear growth-factor spectrum for a proposed ignition double-shell target for the $\mathrm{Na}$ tional Ignition Facility (NIF) and apply a standard saturation analysis to assess the effects of small-scale mix on performance. We find that the target still ignites according to this saturation analysis. The related issue of wave number cutoff for Rayleigh-Taylor and Richtmyer-Meshkov growth is also discussed and we argue that atomic transport effects provide an arguably realistic cutoff for the shortest wavelengths. We conclude in Sec. IV.

\section{ANALYSIS}

We consider a spherical geometry with an interface separating two fluids at $r=R(t)$. The interior fluid is denoted by the subscript " 1 " and the exterior fluid by " 2 ." We introduce a velocity potential $\Psi$ so that the local fluid velocity generally follows from $\mathbf{v}=-\nabla \Psi$. We restrict the analysis to the case where the density $\rho$ on either side of the interface can vary arbitrarily in time but is uniform in space. The continuity equation then reduces to the form $\nabla^{2} \Psi=\dot{\rho} / \rho$, where overdots denote differentiation with respect to time. The general solution of this form of the continuity equation consists of the particular solution plus solutions to Laplace's equation, giving

$$
\begin{aligned}
& \Psi_{1}=\frac{r^{2}}{6} F_{1}+b_{1} r^{\ell} Y_{\ell m}, \\
& \Psi_{2}=\frac{r^{2}}{6} F_{2}+\frac{R^{3}}{3 r}\left(F_{2}-F_{1}\right)+b_{2} r^{-\ell-1} Y_{\ell m},
\end{aligned}
$$

where $F_{i} \equiv \dot{\rho}_{i} / \rho_{i}$, and $b_{i}$ are coefficients to the spherical harmonic $Y_{\ell m}(\theta, \phi)$ of order $\ell$ with $|m| \leqslant \ell$. In Eqs. (1a)(1b) we have imposed the requirement that the perturbation contributions $\left(\propto Y_{l m}\right)$ to the radially symmetric potential decrease away from the interface and that the interior solution be regular at the origin. The coefficients $b_{i}$ are determined from the requirement that the component of velocity normal to the interface be continuous across the (perturbed) interface. The position of the interface is denoted by $r_{s} \equiv R(t)$ $+a(t) \cdot Y_{\ell m}$, where $a \ll R$ is assumed at all times. To zeroth order in $a / R$ we find that $F_{1}=-3 \dot{R} / R$ which enforces mass conservation of the interior fluid. ${ }^{8}$ We emphasize that the form of Eq. (1a) is well-behaved at the origin by design, in contrast to many previous treatments which included a term with an unphysical $1 / r$ dependence. Moreover, the resulting interior fluid radial velocity profile in zeroth order in $a / R$ is strictly linear in $r$, in close agreement with the results of detailed hydrodynamic simulations.

Applying continuity of the normal component of velocity at the interface $\left(r=r_{s}\right)$ we obtain to first order in $a / R$ for the interior and exterior potentials,

$$
\begin{aligned}
\Psi_{1}= & -\frac{r^{2} \dot{R}}{2 R}+\frac{r^{\ell}}{\ell R^{\ell-1}}\left(\frac{a \dot{R}}{R}-\dot{a}\right) Y_{\ell m}, \\
\Psi_{2}= & \frac{R^{3}}{3 r}\left(\frac{3 \dot{R}}{R}+F_{2}\right)+\frac{r^{2}}{6} F_{2} \\
& +\frac{R^{\ell+2} r^{-\ell-1}}{\ell+1}\left(\dot{a}+F_{2} a+\frac{2 \dot{R} a}{R}\right) Y_{\ell m} .
\end{aligned}
$$

However, we note from Eqs. (2a) and (2b) that the tangential component of the fluid velocity is of order $a / R$ and is discontinuous across the interface in general. Thus, the vorticity $(\boldsymbol{\nabla} \times \mathbf{v})$ of the potential flow is nonzero (and unbounded) at the interface, vanishing everywhere else as required.

In addition we must also ensure pressure continuity at the interface using Bernoulli's integral, i.e.,

$$
\begin{aligned}
P_{1}+\rho_{1}\left[\dot{\Psi}_{1}-\frac{1}{2}\left|\nabla \Psi_{1}\right|^{2}\right]_{r=r_{s}} \\
=P_{2}+\rho_{2}\left[\dot{\Psi}_{2}-\frac{1}{2}\left|\nabla \Psi_{2}\right|^{2}\right]_{r=r_{s}},
\end{aligned}
$$

where $P_{1}(t)$ and $P_{2}(t)$ are constants of integration. Using Eqs. (2a) and (2b) in Eq. (3) and evaluating $\left|\nabla \Psi_{i}\right|^{2}$ to first order in $a / R$ we finally obtain to zeroth order and first order in $a / R$, respectively,

$P_{1}(t)-\frac{\rho_{1}}{2} R \ddot{R}=P_{2}(t)+\frac{\rho_{2}}{2}\left(2 R \ddot{R}+3 \dot{R}^{2}+R^{2} \dot{F}_{2}+R \dot{R} F_{2}\right)$,

$\ddot{a}+\frac{\dot{a} \dot{R}}{R}(3-\alpha) \beta_{\ell}-\frac{a}{R}\left[\ddot{R}\left(A_{\ell}(\ell-1)+\alpha \beta_{\ell}\right)+\dot{\alpha} \dot{R} \beta_{\ell}\right]=0$.

Here, $\alpha \equiv-R F_{2} / \dot{R}=-(R / \dot{R})\left(\dot{\rho}_{2} / \rho_{2}\right)$ is a dimensionless shell $(i=2)$ compressibility parameter,

$$
\beta_{\ell}=\frac{\ell \rho_{2}}{\ell \rho_{2}+(\ell+1) \rho_{1}}
$$

and

$$
A_{\ell}=\frac{\ell \rho_{2}-(\ell+1) \rho_{1}}{\ell \rho_{2}+(\ell+1) \rho_{1}}
$$

is a modal Atwood number at the interface. Equation (4b) for the perturbation evolution is the fundamental equation of our model with the middle term responsible for Bell-Plesset 
growth. The strictly geometric origin of this growth is easily seen by neglecting the term (in square brackets) responsible for Rayleigh-Taylor and Richtmyer-Meshkov growth and solving for $\dot{a}$, giving $\dot{a} \propto R^{(\alpha-3) \cdot \beta}$ for $\alpha$ and $\beta$ assumed constant. In the incompressible limit $(\alpha=0)$ and taking $\beta=1$, we have $\dot{a} \propto 1 / R^{3}$ which gives $a \propto 1 / R^{2}$ for $\dot{R} \approx$ const. This result can also be heuristically obtained by considering a $3 \mathrm{D}$ interfacial perturbation of height $a$, wavelength $\lambda=2 \pi R / \ell$, width $\lambda / 2$ and invoking mass conservation. The growth is physically identified with the strong variation of the perturbation wavelength with radius $(\lambda \propto R)$ and the requirement of accommodating an ever decreasing wavelength perturbation on the converging shell. For a compressible or constant thickness shell $(\alpha=2)$ with $\beta=1$ and $\dot{R} \approx$ const, we find from Eq. 4(b) that the perturbation grows only logarithmically with radius, $a=a_{0}+\left(\dot{a}_{0} / \dot{R}\right) \cdot R_{0} \ln \left(R_{0} / R\right)$. By comparison, the heuristic model applied to this compressible case predicts no growth. The mild difference in calculated growth is likely attributed to the neglect of transverse flow in the simple model. However, in both cases the influence of compressibility is predicted to significantly lower the Bell-Plesset growth.

We first compare Eq. (4b) with former work. In the limit of an incompressible shell Eq. (4b) reduces to

$$
\ddot{a}+\frac{3 \dot{a} \dot{R}}{R} \beta_{\ell}-\frac{a \ddot{R}}{R} \cdot(\ell-1) \cdot A_{\ell}=0 .
$$

By contrast, Plesset's governing differential equation (in the limit of vanishing surface tension) reads $^{2}$

$$
\ddot{a}+\frac{3 \dot{a} \dot{R}}{R}-\frac{a \ddot{R}}{R} \cdot\left[(\ell-1) \cdot A_{\ell}-\frac{3 \rho_{1}(\ell+1)}{\ell \rho_{2}+(\ell+1) \rho_{1}}\right]=0 .
$$

Significant differences between the two treatments are apparent. First, the Plesset analysis involves an extraneous term on the left-hand side of Eq. (6b) which may mitigate or aggravate instability according to the value of the density ratio $\beta_{\ell}$. A more important difference involves the term responsible for the Bell-Plesset effect, i.e., the middle term on the left-hand side of Eqs. (6a) and (6b). In the limit of large $\rho_{2}$ both equations are identical. However, the opposite limit shows contrasting behavior between the two analyses. For example, Plesset has claimed that an instability exists even in the (Rayleigh-Taylor stable) case when $\ddot{R}>0$, provided $(2 \ell+1) R \ddot{R}<(3 / 2) \dot{R}^{2}{ }^{2}$ By contrast Eq. (6a) shows that the middle term responsible for growth in the limit of large $\rho_{1}$ vanishes altogether. Thus, an expanding $(\dot{R}>0)$ and accelerating $(\ddot{R}>0)$ high-density $\left(\rho_{1} \gg \rho_{2}\right)$ bubble is not unstable within the limits of this linear perturbation analysis. The basic difference between the two treatments is that Eq. (6a) is associated with conservation of interior mass, whereas Eq. (6b) requires a source or sink at the origin according as $\dot{R}$ is positive or negative.

We now return to Eq. (4b) to discuss the effect of shell compressibility. Because the Bell-Plesset effect is directly attributed to the incompressibility of a converging shell, finite compressibility will act to reduce the size of the effect.
For example, in the thin shell approximation, ${ }^{10}$ where the thickness $\Delta$ of the imploding shell is small compared to the radius of the shell, $\alpha=2+(R / \dot{R}) \cdot(\dot{\Delta} / \Delta)$. In the incompressible limit $(\dot{\rho} \equiv 0), \dot{\Delta} / \Delta=-2 \dot{R} / R$ and $\alpha=0$. In the compressible case $(\dot{\Delta} / \Delta=0)$ under constant drive conditions, $\alpha=2$. For most cases of interest in ICF the imploding shell satisfies $\alpha=2$ to a good approximation up to the time of deceleration onset. Thereafter, the stagnation pressure of the fuel rises rapidly and further compression of the shell beyond the effect of spherical convergence may result. This additional contribution to shell compressibility can be estimated from momentum balance, assuming a polytropic equation of state $P_{2} \rho_{2}^{-\gamma}=$ const for the shell, giving

$$
\Delta=\frac{1}{\rho_{2}}\left|\frac{P_{2}}{\ddot{R}}\right| \propto P_{2}^{-1 / \gamma},
$$

where mass ablation is neglected and the deceleration $(\ddot{R}$ $<0$ ) during shell stagnation is taken to be proportional to the pressure $P_{2}$. To evaluate $\dot{\Delta} / \Delta$ from Eq. (7) we require the time scale for pressure stagnation $\tau_{s}>0$ which we take as the difference between deceleration onset $(\ddot{R}=0)$ and stagnation $(\dot{R}=0)$. Applying energy conservation we obtain ${ }^{11}$

$$
\tau_{s}=-\frac{R_{d}}{\dot{R}_{d}}\left[1-\left(\frac{R_{d}}{R_{s}}\right)^{2}\right],
$$

where $R_{d}$ is the inner radius of the shell at deceleration onset and $R_{s}$ is the minimum (or stagnation) radius of the shell. We can now write for $\alpha$ after deceleration onset

$$
\alpha=2+\frac{R}{\dot{R}} \frac{\dot{\Delta}}{\Delta} \approx 2-\frac{R_{d}}{\dot{R}_{d}} \cdot \frac{1}{\gamma \tau_{s}}=2+\frac{1}{\gamma \cdot\left[1-\left(\frac{R_{s}}{R_{d}}\right)^{2}\right]} .
$$

For most implosions of interest, $\left(R_{s} / R_{d}\right)^{2} \leqslant 0.1$ so that $\alpha$ $\approx 2+1 / \gamma$ to a good approximation. Thus, we expect $\alpha$ to generally lie in the range of 2-3 after deceleration onset which is corroborated by simulation studies. Referring back to Eq. (4b) we indeed find that the effect of compressibility to a large degree cancels the effect of spherical convergence and provides a significant reduction in the Bell-Plesset effect. In the following we verify this result by directly applying our analysis to a proposed double-shell ignition target for the NIF.

\section{APPLICATION TO DOUBLE-SHELL IGNITION TARGET DESIGNS}

\section{A. Linear growth factor analysis}

An important application of the analysis described above is towards a proposed class of ignition double-shell target for the NIF (see Fig. 1). ${ }^{11}$ A key concern for this type of target is the buildup of short wavelength perturbations on the inner surface of the high- $Z$ inner shell that can lead to deleterious mixing of cool high- $Z$ shell material and hot burning fuel. Analysis suggests that most of the instability growth is due to the Rayleigh-Taylor instability following deceleration onset. Richtmyer-Meshkov instability is important before de- 


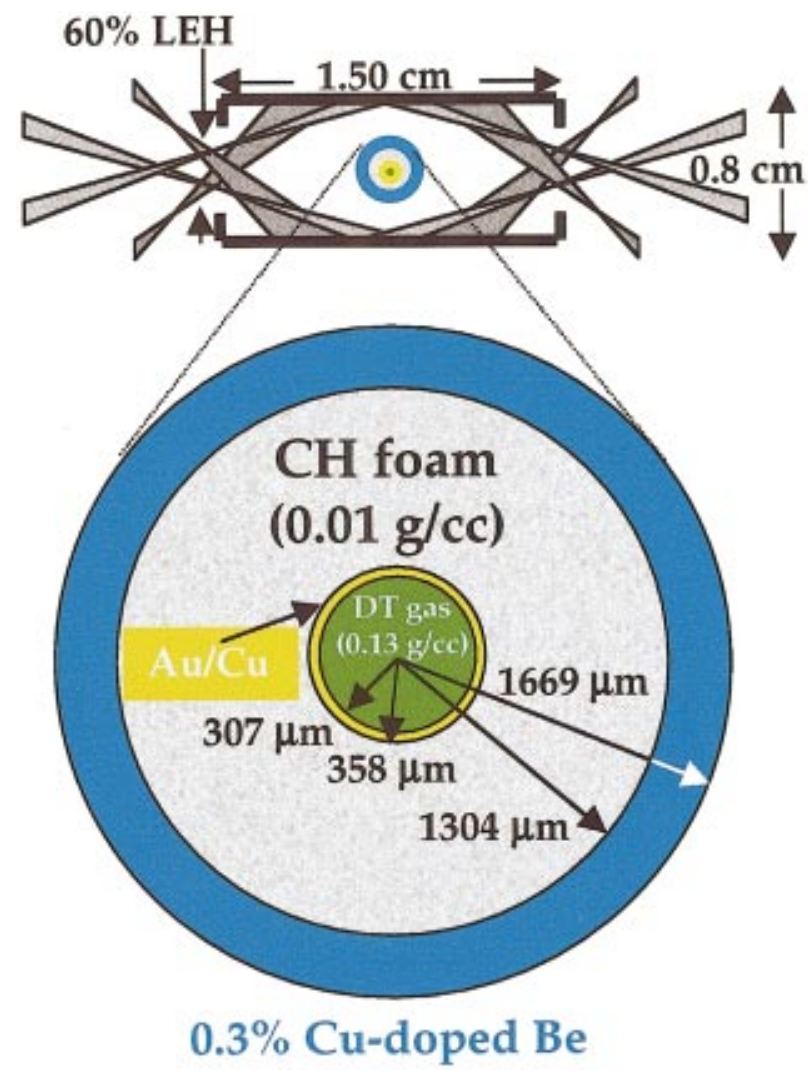

FIG. 1. (Color) Schematic of NIF double-shell ignition target that absorbs $750 \mathrm{~kJ}$ of x-ray energy with an incident laser energy of $2.5 \mathrm{MJ}$ driving a hohlraum at a drive temperature of $250 \mathrm{eV}$ over $10 \mathrm{~ns}$. Inner and outer laser cones shown with $60 \%$ laser-entrance-holes.

celeration onset and acts as a seed for the far more dominant Rayleigh-Taylor growth. The usual expression for the Rayleigh-Taylor growth rate under ICF conditions, neglecting convergence and compressibility effects, is given by the following: ${ }^{7}$

$$
\gamma=\sqrt{\frac{A k \ddot{R}}{1+A k L}}-\beta k v_{a},
$$

where $A \equiv\left(\rho_{2}-\rho_{1}\right) /\left(\rho_{2}+\rho_{1}\right)$ is the Atwood number of the unstable interface, $k$ is the perturbation wavenumber, $L$ is a density-gradient scale length across the interface, $\beta$ is a phenomenological constant between 1 and 3 , and $v_{a}$ is the ablation velocity. On the pusher-fuel interface of the proposed double-shell target, the materials are distinct and $L$ is identically zero in the absence of mix or diffusion, i.e., finite density-gradient stabilization in the linear growth regime does not occur. The effect of mass ablation of the high- $Z$ inner shell is also negligible so that ablation stabilization is minimal. Finally, $A \approx 1$, i.e., $\rho_{2} \gg \rho_{1}$, so that classical Rayleigh-Taylor growth is expected to occur, i.e., $\gamma$ $\cong \sqrt{k \ddot{R}}$. Thus, we conclude that virtually no stabilization of high mode-number perturbations can be expected on the pusher-fuel interface of a double-shell according to Eq. (10).

A more appropriate estimate for Rayleigh-Taylor growth in double-shell targets may be found in the treatment of Duff, Harlow, and Hirt where the effects of mass and velocity diffusion are included, ${ }^{12}$

$$
\gamma=\sqrt{\frac{A k \ddot{R}}{\eta}+\nu^{2} k^{4}}-\left(\nu+D_{12}\right) k^{2} .
$$

Here, $\eta(k, t)$ is a growth-rate reduction factor due to a timevarying binary mass diffusion layer of thickness $\delta_{D}$ $=2 \sqrt{D_{12} t}$ at the interface, $\nu$ is the kinematic viscosity, and $D_{12}$ is the binary mass diffusivity. The combined effects of viscosity and binary mass diffusion will now introduce a cutoff in Rayleigh-Taylor growth at sufficiently high wavenumber in marked contrast to Eq. (10) in the absence of ablative stabilization. In Sec. III C we return to Eq. (11) to discuss in more detail the stabilizing effect of mass diffusion and viscosity for the proposed double-shell ignition target.

Our goal in this section is to extract a linear growthfactor spectrum for the double-shell ignition design including the combined effects of spherical convergence, shell compressibility, Rayleigh-Taylor instability, and RichtmyerMeshkov instability. In Sec. III B we will implement this growth-factor spectrum in a mode saturation analysis to estimate the amount of yield degradation arising from the effects of pusher-fuel mix. Ordinarily, the growth-factor spectrum for mainline ICF targets is straightforwardly obtained from a suite of 2D single-mode simulations. However, we find that carrying through a single-mode growth-factor simulation of double-shells at high mode numbers of interest is a particularly daunting exercise. To date we have not succeeded in performing a credible simulation at even relatively low mode numbers because of the vexing task of ensuring numerical stability while not compromising the integrity of the simulation with excessive numerical filtering. A semianalytic option is to implement the analytical treatment described in Sec. II using detailed 1D simulations for the radiation-hydrodynamical history of the imploding shell, e.g., position, speed, acceleration, Atwood number, and compressibility. In particular, a radiation-hydrodynamics simulation for the zeroth-order shell dynamics supersedes Eq. (4a), and the same unperturbed shell quantities are used in Eq. (4b) to drive the perturbation growth. An important advantage of this procedure is that a very detailed radiationhydrodynamical description of the imploding shell is possible in $1 \mathrm{D}$-far more than is currently practical with a $2 \mathrm{D}$ growth-factor simulation.

We have applied this semi-analytic methodology to the double-shell design depicted in Fig. 1. This design is intended to have minimal feed through of outer-surface perturbations to the inner surface of the high- $Z$ shell as well as reduced Rayleigh-Taylor growth of intrinsic surface perturbations on the inner surface. We first show 1D simulated histories of various zeroth-order shell quantities which will then be used in Eq. (4b). Figure 2(a) shows the Atwood number history on the pusher-fuel interface up to the instant of peak energy production. We find that the Atwood number is considerably reduced from its initial value, reaching close to 0.5 near peak compression. Compared to a $2 \mathrm{D}$ growth factor simulation with standard zoning, this value is somewhat lower $(\approx 10 \%)$ and underscores an important advantage in using 1D simulations for a convenient and accurate assessment of hydrodynamic phenomena. Away from the interface 

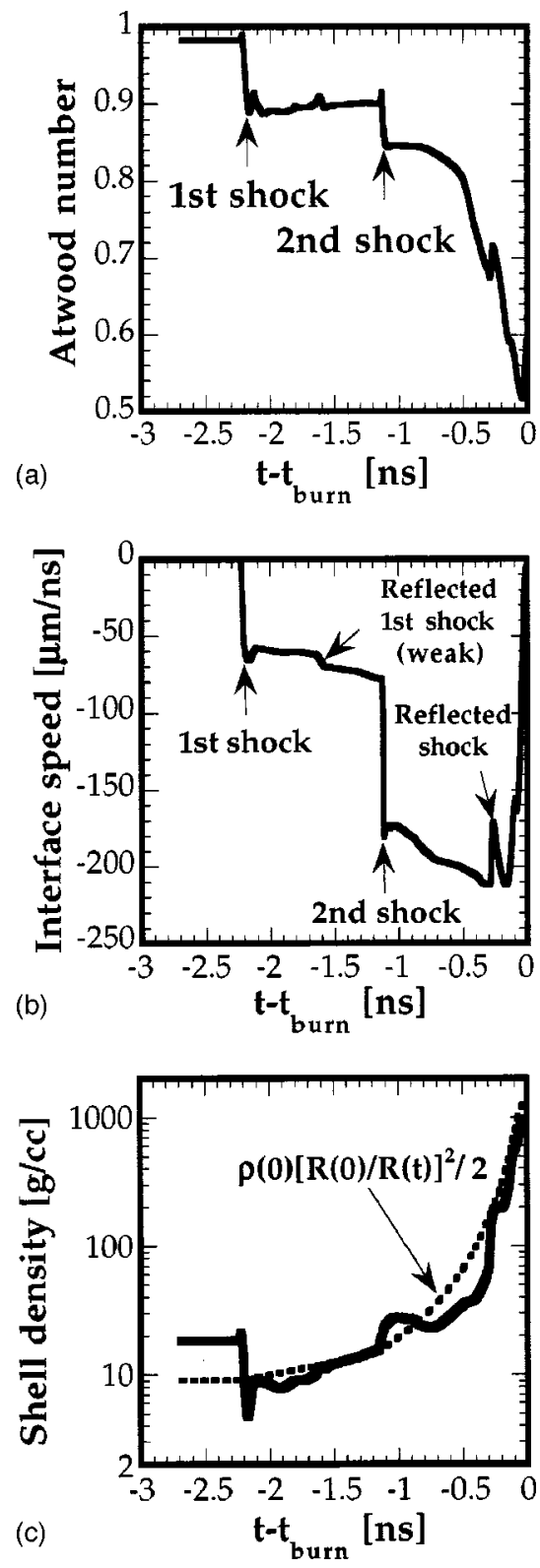

FIG. 2. Interface Atwood number (a), speed (b), and shell density near interface (c) vs time relative to peak energy production for NIF double-shell ignition target (Fig. 1).

the Atwood number approaches unity very quickly due to the rapid increase in shell density with radius. Low mode number perturbations have an appreciable radial extent and can sample such higher values of Atwood number. Our focus here is on high mode number perturbations due to their intrinsically large growth rates. Such modes have radial extent $\approx R / \ell$ which is on the order of 0.2 microns or less for mode numbers greater than 100 near ignition. From simulation studies the change in Atwood number over this distance is less than $6 \%$ which results in an underestimate of the growth-rate by less than $3 \%$. In the following we will evaluate growth-factors based on the interface Atwood number, thereby ignoring this small yet calculable correction for simplicity. Figure 2(b) shows the velocity history of the pusherfuel interface and the presence of two principal shocks that drive the implosion. Figure 2(c) furnishes information on the compressibility of the imploding shell. The solid curve depicts the simulated density of the inner shell very close to the pusher-fuel interface. Transient effects from shock and rarefaction passage are quite evident and tend to complicate quantitative identification of the trend in shell compressibility. Moreover, the compressible model described above (see Sec. II) is based on the assumption of uniform density profiles on both sides of the interface and, as such, has no license to describe transient or localized density effects. To extract the gross behavior of the shell compressibility we have overlaid the simulation curve with a parameterization of the shell density in terms of the shell convergence only,

$$
\rho(t)=(\rho(0) / 2) \cdot[R(0) / R(t)]^{2},
$$

where $R(t)$ is the simulated trajectory of the interface. We see that the simulated density follows a $R(t)^{-2}$ behavior fairly well over the course of the implosion, suggesting that the shell behaves compressibly to a high degree. This implies that $\alpha \cong 2$ with $\dot{\alpha} \cong 0$ on average, leading to a significant reduction of the Bell-Plesset effect, cf. Eq. (4b). The stabilizing effect of slow compression on the linear stability of an accelerated shear layer has been shown earlier in the case of slab geometry. ${ }^{13}$

We now turn to the task of calculating the perturbation growth on the pusher-fuel interface for the above-described implosion using Eq. (4b). Figure 3(a) shows the growthfactor history for a perturbation with mode number $\ell=100$. Until the onset of deceleration the growth-factor history is dominated by Richtmyer-Meshkov growth. The various phase reversals evident in Fig. 3(a) can be understood from the analytic expression for ideal Richtmyer-Meshkov growth,

$$
\mathrm{GF}=1+A \cdot \Delta v \cdot \Delta t \cdot \ell / R,
$$

where GF is the growth-factor, $\Delta v$ is the change in interface speed induced by passage of a shock, $\Delta t$ is the duration of constant interface speed, and $A$ is understood to represent the postshock Atwood number. For simplicity, we have elected not to distinguish between the preshock and postshock amplitudes in Eq. (13). Because $\Delta v$ is generally less than zero for an imploding shell, the growth factor undergoes a sign change whenever $A \cdot|\Delta v| \cdot \Delta t \cdot \ell / R>1$. With a series of shocks and reflected shocks affecting the interface motion in a double-shell, the cumulative effect of the RichtmyerMeshkov instability is not large due to cancellation from successive phase reversals. Still, the modest amplitude remaining at deceleration onset can provide a significant seed for the subsequent and far stronger Rayleigh-Taylor instability growth.

Figure 3(b) depicts the peak-growth-factor spectrum predicted from Eq. (4b). The peak in growth-factor is defined as the normalized growth at the instant of peak energy production. The top curve represents the spectrum of growth for a spherically converging incompressible shell. This case corresponds to pure Bell-Plesset growth without the mitigating effects of shell compressibility. The intermediate curve includes both the effects of shell compressibility and spherical convergence. The lower curve shows the growth-factor spectrum in the absence of shell compressibility and spherical 

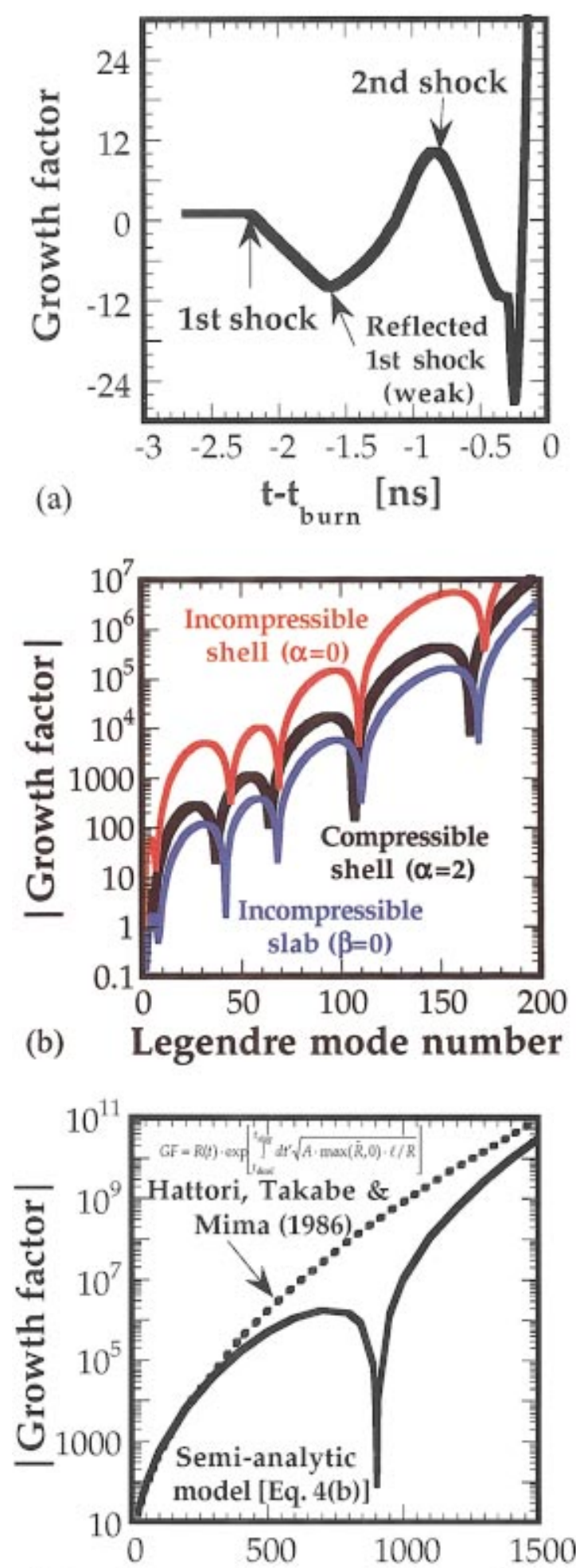

(c)

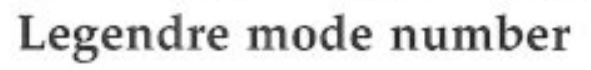

FIG. 3. (Color) (a) NIF double-shell growth-factor vs time for mode number $\ell=100$ based on the semianalytic model [Eq. 4(b)]; (b) calculated growthfactor spectrum at peak thermonuclear burn from semianalytic model for three cases: Bell-Plesset effect without shell compressibility (red), BellPlesset effect with shell compressibility (black), without Bell-Plesset effect and without shell compressibility (blue); (c) comparison of growth-factor spectrum at peak burn from the semianalytic model [Eq. 4(b)] and approximate model of Hattori, Takabe, and Mima (Ref. 14) calculated from deceleration onset to instant of peak energy production. convergence. Overall, we find that shell compressibility reduces the growth-factor by nearly an order-of-magnitude in the presence of spherical convergence, i.e., the Bell-Plesset effect. Compared with the simplest case of an incompressible shell and no spherical convergence, i.e., the lower curve in Fig. 3(b), the combined effect of Bell-Plesset effect with shell compressibility gives about one $e$-folding more growth. All three curves show the presence of spectral lobes which are attributable to phase reversal from Richtmyer-Meshkov growth. When the condition $\ell \approx R(t) /(A \cdot|\Delta v| \cdot \Delta t)$ is satisfied for any Richtmyer-Meshkov episode [see Fig. 3(a)] then the seed amplitude for Rayleigh-Taylor growth after deceleration onset is expected to be small. Over the range of mode numbers shown in Fig. 3(b) the maximum growth-factor for each lobe in the compressible Bell-Plesset case is found to scale closely as $\mathrm{GF} \sim \exp \left(\ell^{0.53}\right)$ which is somewhat larger than the classical Rayleigh-Taylor scaling $\mathrm{GF} \sim \exp (\sqrt{\ell})$ at high mode number.

Figure 3(b) also indicates a shift in the location of the spectral lobes as a function of shell compressibility. We can gain a quantitative understanding of this effect from studying Eq. (4b) and treating the middle term as a perturbation. Accordingly, Eq. (13) is modified by compressibility as follows:

$$
G F=1+A \cdot \Delta v \cdot \Delta t \cdot(\ell / R) \cdot\left[1-\frac{\Delta v \cdot \Delta t \cdot(3-\alpha)}{2 R}\right]
$$

On physical grounds, greater Richtmyer-Meshkov growth can be expected for an incompressible shell because shell thickening promotes a delay in arrival of the next shock at the interface, effectively leading to a larger growth duration $\Delta t \propto \Delta(t) / v_{s} \propto\left[\Delta_{0} / R^{2}(t)\right]$, where $v_{s} \propto 1 / \sqrt{\rho_{2}}$ is the shock speed. By comparison, the delay for a compressible shell $(a=2)$ has a weaker scaling with shell radius, $\Delta t \propto \Delta_{0} / v_{s}$ $\propto \Delta_{0} \sqrt{\rho_{2}} \propto 1 / R(t)$. Evaluating the last term on the right-hand side of Eq. (14) for the effect of compressibility gives a correction on the order of $5 \%-10 \%$ to the growth-factor for the earliest shock depicted in Fig. 2(b). To find the location of a spectral lobe we set the right-hand side of Eq. (14) to zero and solve for the mode number,

$$
\ell=-\frac{R}{A \cdot \Delta v \cdot \Delta t} \cdot \frac{1}{\left[1-\frac{\Delta v \cdot \Delta t}{2 R}(3-\alpha)\right]}
$$

With $\Delta v<0$ and $\Delta v \Delta t / R$ on the order of 0.1 , we find a shift in mode number due to shell compressibility ( $\alpha=2$ vs $\alpha=0$ ) of nearly $-10 \%$, in agreement with the trend seen in Fig. 3(b).

For completeness we compare with former work using different techniques. Hattori, Takabe, and Mima have applied self-similar methods for studying Rayleigh-Taylor instability in a spherically stagnating system, obtaining the following approximate expression for adiabatic perturbation growth: ${ }^{14}$

$$
a_{\ell}(t) \propto R(t) \cdot \exp \left[\int^{t} d t^{\prime} \sqrt{A\left(t^{\prime}\right)|\ddot{R}| \ell / R\left(t^{\prime}\right)}\right] .
$$

In Fig. 3(c) we compare Eq. (16) with our semianalytic model [based on Eq. 4(b)] for the NIF double-shell ignition 
design during the deceleration phase only. At mode numbers below $\ell=400$ the two treatments are seen to agree to within a factor-of-2. At larger mode numbers the effect of Richtmyer-Meshkov instability from a reflecting shock becomes evident, leading to a significant difference at mode numbers $\ell<1500$. In the near absence of ablative stabilization these short wavelengths are important for double-shell stability as we explore in more detail in Sec. III C.

\section{B. Mode saturation analysis}

At high mode number a growing perturbation quickly reaches an amplitude on the order of its wavelength at which time saturation effects begin to dominate. The Haan saturation criterion describes the threshold amplitude $S$ for the onset of nonlinear effects in the presence of a full spectrum of modes, ${ }^{6}$

$$
S(R, \ell)=\frac{2 R}{\ell^{2}} .
$$

The Haan model does not explicitly treat nonlinear effectsonly to the extent that modes are assumed to grow linearly in time once the (nonlinear) saturation threshold is met. A model which includes both pre- and postsaturation mode coupling has been developed by Ofer et al. ${ }^{15}$ The methodology for applying the Haan saturation analysis is as follows. An initial surface roughness spectrum is convolved with a time-dependent linear growth-factor spectrum to determine the onset of saturation for each mode. A quadrature sum of each growing mode, whether still in the exponential or linear stage of growth, is then formed to find an overall mix width vs time. This mix width is represented in terms of bubble and spike components where the spike amplitude is related to the bubble amplitude by the approximate factor $1+A$. The dynamic mix layer is then used in a $1 \mathrm{D}$ simulation to assess its effect on yield degradation assuming full atomic mixing of fuel and pusher material throughout the mixing layer. This procedure has been successfully applied to a wide variety of ICF targets that have been fielded on the Nova and Omega lasers over recent years. ${ }^{16,17}$ The appropriateness of the Haan prescription is optimally met under weakly nonlinear conditions such as when ablative stabilization severely limits the growth of large wavenumber modes. In contrast, the doubleshell target undergoes highly nonlinear perturbation growth on the inner surface of the pusher where ablation stabilization is minimal. Still, this property does not invalidate application of the Haan prescription as a tool to estimate the effect of pusher-fuel mix on target performance. In the presence of a dense spectrum of modes the saturation criterion remains intact but the assumption of subsequent linear growth will not hold under conditions of strong mode coupling.

Figure 4 shows the initial mode spectrum of a typical ICF plastic capsule fielded on the Omega laser. We adopt this surface spectrum as a crude surrogate for the expected $\mathrm{Au}$ surface finish of the proposed double-shell ignition target (Fig. 1). We now apply the Haan prescription. By the time of deceleration onset Fig. 4 shows the degree of modal growth with the smallest wavelength modes undergoing the greatest growth as expected. At peak burn the amplitudes of the high- est modes have hardly changed due to saturation effects but the low and moderate modes continue to show the largest growth. The quadrature sum of the modes $\left(\sigma^{2}\right)$ shown at peak burn corresponds to a spike amplitude $[\sqrt{2}(1+A) \cdot \sigma]$ of only 5 microns compared to a converged fuel radius of 25 microns. The thermonuclear yield from this target still gives $1 \mathrm{MJ}$ compared to a clean (undegraded) yield of $2.8 \mathrm{MJ}$. Thus, the ignition double-shell target design still ignites despite the level of mix predicted by the Haan analysis.

Although the assumption of linear mode growth following saturation may not be strictly valid for double-shells, the prescription has an important advantage over other methods for evaluating nonlinear mix, e.g., Ramshaw's nonlinear mix model $^{18}$ and the $\mathrm{K}-\mathrm{L}$ turbulence mix model. ${ }^{19}$ Generally these methods rely on a set of phenomenologically constrained parameters for implementation. However, the Haan analysis is largely independent of such parametric constraints with one possible exception: an enhanced heat diffusivity term which is meant to mimic in 1D the extra heat dilution arising from a highly modulated interface. ${ }^{16}$ We find that the amount of thermonuclear yield in the double-shell ignition design is only weakly sensitive to the size of this diffusivity term, thereby largely preserving the parametric independence of the analysis.

We have made no distinction in our analysis between saturation of the Bell-Plesset or geometric component of perturbation growth and saturation of the usual dynamical components, e.g., Richtmyer-Meshkov and RayleighTaylor. This assumption is not likely to be valid, but its attendant error is probably negligible owing to the comparatively small effect of geometric growth for the problem at hand. Work is in progress to understand this aspect of BellPlesset behavior by extending the analysis of Sec. II to second order in $a / R$ and exploring the possibility of geometric mode-coupling.

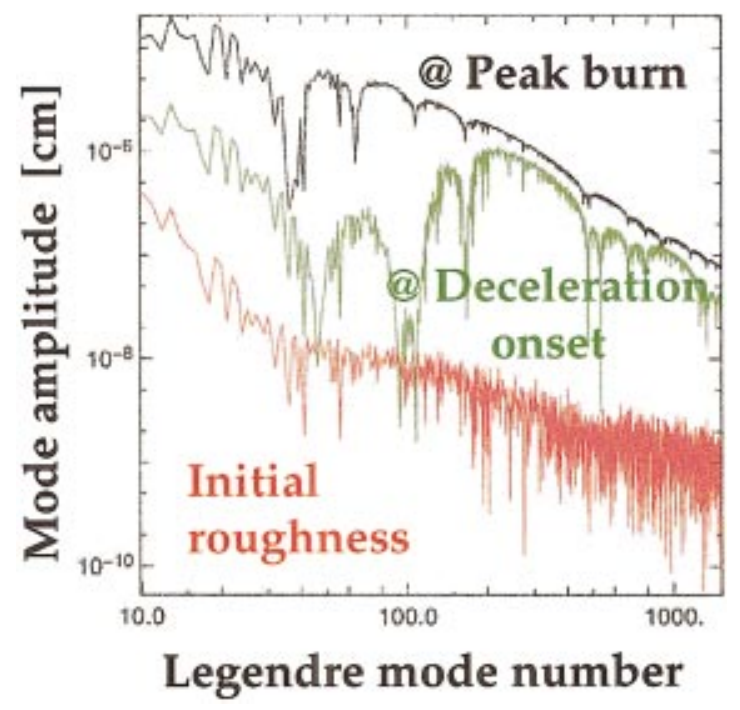

FIG. 4. (Color) Initial mode spectrum (red), mode spectrum at deceleration onset (green), and mode spectrum at peak burn (black) according to Haan saturation analysis (Ref. 6) of NIF double-shell ignition target (Fig. 1). 


\section{Rayleigh-Taylor mode cutoff}

In applying the Haan analysis we have considered modes up to $\ell=1500$. From a formal standpoint, the calculated mix width is mathematically bounded, ${ }^{6}$ converging weakly with cutoff mode number $\ell_{c}$ as $\sigma \propto \ell_{c}^{-1 / 2}$. Although the results are seen to be insensitive to the maximum mode number used over this range, we still need to place reasonable physical bounds on the mode numbers that matter to the problem in anticipation of detailed multimode simulations. To this end we return to Eq. (11) for further analysis. The kinematic viscosity $\nu$ is calculated according to the method of Clerouin et $a l .{ }^{20}$ which was based on molecular dynamics simulations in the dense plasma regime and extrapolation to the dilute plasma regime. This model is applicable over a wider range of temperature and density than the familiar Braginskii model. ${ }^{21}$ The plasma binary mass diffusivity $D_{12}$ is based on the method of Paquette et al., ${ }^{22}$ where a rigorous derivation in the dilute plasma regime is carried out and then extrapolated to the dense plasma regime. Molecular dynamics simulations are used to verify the analysis in the regime of intermediate plasma coupling characteristic of double-shells. The diffusivity is used to evaluate the growth-rate reduction factor $\eta[(\ell / R), t]$ arising from a relaxation of the density gradient across the atomically mixed interface. This factor is found by solving for the eigenvalue of a second order equation for the velocity perturbation normal to the mean interface using a prescribed form for the interfacial density profile, $\rho=\operatorname{erf}\left[R(t) / \sqrt{4 D_{12} t}\right]$; details of this procedure are described elsewhere. ${ }^{21}$

We have evaluated Eq. (11) vs time on both sides of the Rayleigh-Taylor unstable interface for the double-shell ignition design (see Fig. 1). We expect that the largest value in wavenumber cutoff occurs at deceleration onset when $D_{12}$ is relatively small and the effects of density-gradient stabilization, i.e., $\eta(k, t)$, and static diffusion stabilization, $-D_{12} k^{2}$, are still minimal. Furthermore, we also anticipate that the pusher-side of the interface will be more susceptible to high mode number instability due to the much lower kinematic viscosity of Au compared with the fuel-side of the interface. Figure 5(a) shows the Rayleigh-Taylor growth rate at deceleration onset vs mode number [Eq. (11)] on the Au side of the interface including various atomic transport effects as indicated. The top curve represents classical RayleighTaylor growth in the absence of ion viscosity, atomic diffusion, and density-gradient stabilization. The next lower curve isolates the addition of ion viscosity and attains a maximum near $\ell=10^{4}$ (not shown). The further inclusion of static diffusion stabilization is an important contribution as illustrated in Fig. 5(a), giving a cutoff in mode number at $\ell \approx 8000$. Finally, the additional effect of density gradient stabilization is represented by the lowest curve, indicating a cutoff in mode number of $\approx 3700$. We also note that the maximum growth occurs near $\ell \approx 1200$ and is already reduced by a factor-of-2 from the classical Rayleigh-Taylor growth-rate. The mode number cutoff for Rayleigh-Taylor growth as well as the mode number for which classical Rayleigh-Taylor growth is reduced by a factor-of- 2 are shown as a function of time in Fig. 5(b), starting with deceleration onset and con-
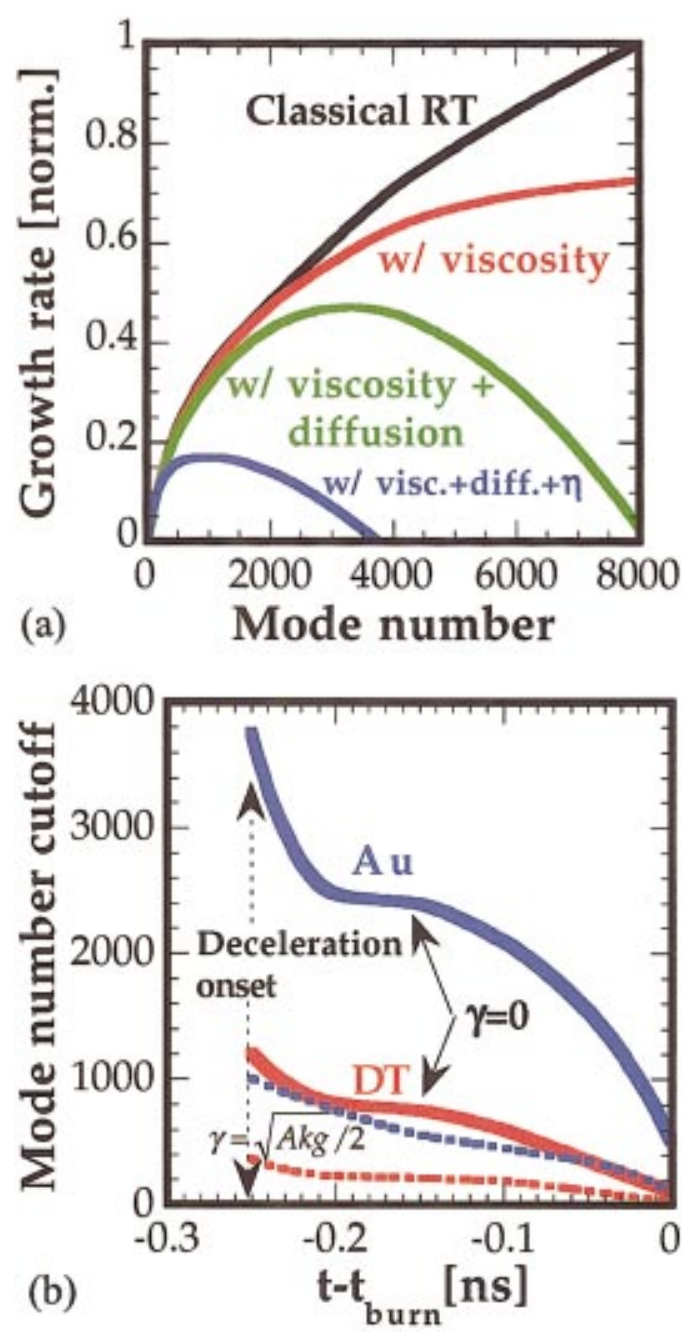

FIG. 5. (Color) (a) Calculated linear normalized growth-rates of NIF double-shell ignition target design (Fig. 1) at deceleration onset according to the model of Duff, Harlow, and Hirt (Ref. 12) for classical Rayleigh-Taylor growth (black curve), including ion viscosity (red curve), including viscosity and static diffusion (green curve), and combined viscosity, static diffusion, and density gradient stabilization (blue curve). (b) Calculated mode numbers for vanishing growth-rate $(\gamma=0)$ vs time on the Au pusher (solid blue curve) and DT fuel (solid red curve) side of interface; calculated mode numbers for the growth-rate reduced to one-half of the classical Rayleigh-Taylor growth-rate $(\gamma=1 / 2 \sqrt{A k g})$ vs time on the Au pusher (dotted blue curve) and DT fuel (dotted red curve) side of the interface.

tinuing to peak burn. As expected, the pusher side of the interface shows a significantly higher mode cutoff compared with the fuel. Increasing viscosity and binary mass diffusion combined with a decreasing length scale lead to a nearly linear decrease in mode cutoff for both sides of the interface as the inner shell converges. From this analysis the maximum cutoff mode number ( $\ell \approx 3700$ ) occurs at deceleration onset and within the high- $Z$ pusher. Of greater interest is the mode number at which the Rayleigh-Taylor growth-rate is reduced by a factor-of-2. This latter mode number attains a maximum near 1200 at deceleration onset and is reasonably close to the cutoff adopted for the above saturation analysis (see Sec. III B).

\section{Richtmyer-Meshkov mode cutoff}

We have argued for a practical mode number cutoff ( $\ell$ $\approx 1200$ ) for Rayleigh-Taylor growth occurring at decelera- 


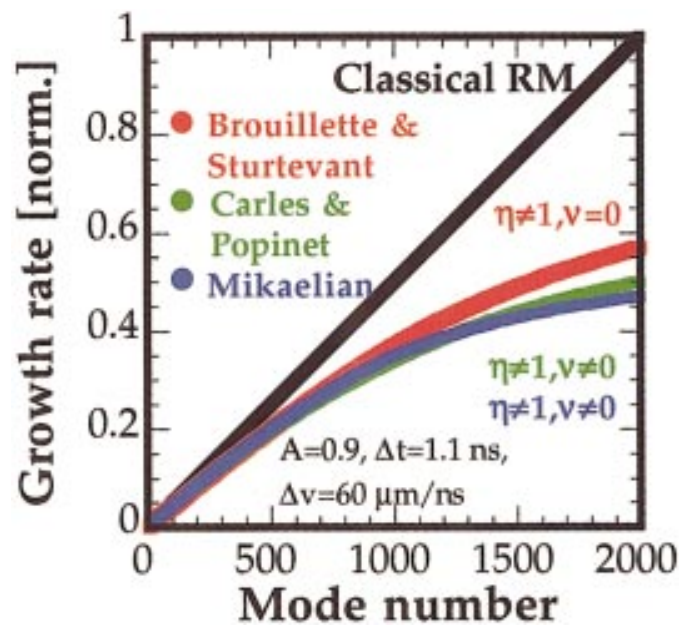

FIG. 6. (Color) Calculated linear normalized growth-rates for the Richtmyer-Meshkov instability from the first shock transit for the NIF double-shell ignition target design (Fig. 1) in the fluid limit (black curve), including mass diffusion (red curve), including mass and momentum diffusion according to models of Carlès and Popinet (Ref. 25) (green curve) and Mikaelian (Ref. 26) (blue curve) for viscosity, and mass diffusion model of Brouillette and Sturtevant (Ref. 21).

tion onset. Although the overall growth of instability in ignition double-shells appears to be greatly dominated by Rayleigh-Taylor, it is of interest to ask how well the above cutoff applies to the prior occurrence of RichtmyerMeshkov growth. To this end we invoke some recent work on the effect of atomic mass and momentum diffusion on the Richtmyer-Meshkov instability.

Brouillette and Sturtevant ${ }^{23}$ have argued for combining the model of Duff et al. ${ }^{12}$ with the impulsive acceleration formulation of Richtmyer ${ }^{24}$ to obtain the following extension of Eq. (13), including atomic mass diffusion,

$$
\dot{a}_{\ell}=a_{\ell}^{\prime} \frac{A \cdot \Delta v \cdot(\ell / R)}{\eta[(\ell / R), t]} .
$$

Here, $\eta$ is the growth-factor reduction factor from binary mass mixing across the interface as before (Sec. III C), and the post-shock amplitude is now distinguished by a primed notation. The effect of binary mass diffusion on our doubleshell ignition design is depicted in Fig. 6 for the case of the first shock episode [see Fig. 3(a)]. Compared to the growthfactor for Richtmyer-Meshkov growth in the fluid limit, we find a near factor-of- 2 reduction from the effects of binary mass diffusion alone near $\ell=2000$.

Carlès and Popinet have recently reexamined the effect of viscosity on the Richtmyer-Meshkov instability based on an asymptotic study of the Navier-Stokes equations using singular perturbation techniques. ${ }^{25}$ The following form for the linear growth-factor spectrum is obtained:

$$
\begin{aligned}
G F= & (1+A \cdot \Delta v \cdot \Delta t \cdot \ell / R)-\frac{16}{3 \pi} \\
& \times \frac{\sqrt{\rho_{1} \mu_{1}} \sqrt{\rho_{2} \mu_{2}}}{\left(\rho_{1}+\rho_{2}\right)\left[\sqrt{\rho_{1} \mu_{1}}+\sqrt{\rho_{2} \mu_{2}}\right]} A \cdot \Delta v \cdot(\ell / R)^{2} \cdot \Delta t^{3 / 2},
\end{aligned}
$$

where $\mu=\rho \nu$ is the dynamic viscosity. In Fig. 6 we have plotted the combined effects of mass and momentum diffusion by adapting Eq. (19) through use of a multiplier $(1 / \eta)$ on the first term on the right-hand side as suggested by the form of Eq. (18). A further reduction in the growth-factor from viscosity is seen with a maximum in the spectrum occurring near $\ell=4000$ (not shown). For comparison we have also plotted the viscous damping model of Mikaelian ${ }^{26}$ but similarly adapted to include the effect of mass diffusion. A similar strong reduction in growth-factor is predicted according to this model as well. Additional reduction can be expected from the effect of static diffusion stabilization as was the case for Rayleigh-Taylor instability (Sec. III C), although this contribution has not been studied in the context of Richtmyer-Meshkov growth to our knowledge. Although a true cutoff in Richtmyer-Meshkov growth from atomic diffusion is not evident over the indicated range of mode numbers shown in Fig. 6, the relatively meager growth suggests that our adopted Rayleigh-Taylor cutoff near $\ell$ $\approx 1200$ is expected to be little affected.

\section{SUMMARY}

We have used a modified form of the velocity potential to revisit the analyses of Bell, ${ }^{1}$ Plesset, ${ }^{2}$ and Fisher $^{4}$ with imposed conservation of mass in the volume interior to the interface of interest. In this manner we have managed to avoid an unphysical divergence in the fluid velocity at the origin. In the limit of small interior density compared to the ambient density, agreement is found between our analysis and previous work. However, important differences arise in the ICF-relevant regime where the interior (fuel) density becomes comparable to the ambient (pusher) density at peak compression. We have applied our model for perturbation growth to a calculation of the growth-factor spectrum for a proposed double-shell ignition target on the NIF. The effect of Richtmyer-Meshkov instability is shown to be important both as a seed for subsequent Rayleigh-Taylor growth and for modulating the growth-factor spectrum. The growthfactor spectrum is used as input to a Haan saturation analysis for estimating the effect of mix on the double-shell target; we find that the target still ignites. The related question of mode cutoff for Rayleigh-Taylor growth is addressed based on a former analysis of the stabilizing effects of ion viscosity and binary mass diffusion. A practical cutoff in high mode number $\ell \approx 1200$ is argued for the proposed double-shell ignition target. Such a manageably low cutoff in mode number is encouraging from the standpoint of ultimately performing a detailed numerical simulation. We envision over the near term the possibility of carrying through a $2 \mathrm{D}$ multimode simulation of the double-shell ignition target to more definitively assess its robustness to nonlinear mix.

\section{ACKNOWLEDGMENTS}

The authors acknowledge useful discussions with M. J. Edwards, S. W. Haan, O. Jones, K. O. Mikaelian, S. M. Pollaine, L. J. Suter, and R. E. Tipton. 
This work was performed under the auspices of the U.S. Department of Energy by the University of California Lawrence Livermore National Laboratory under Contract No. W-7405-Eng-48.

${ }^{1}$ G. I. Bell, Los Alamos Scientific Laboratory Report No. LA-1321, 1951. ${ }^{2}$ M. S. Plesset, J. Appl. Phys. 25, 96 (1954).

${ }^{3}$ G. Birkhoff, Q. Appl. Math. 12, 306 (1954).

${ }^{4} \mathrm{H}$. N. Fisher, "Instabilities in converging compressible systems," LANL memorandum No. X-1(5/82) 22, 1982 (unpublished). An algebraic error in his analysis has led to the erroneous result that convergence effects dominate over Rayleigh-Taylor growth. However, when his Eq. (4.10) is replaced by the following expression: $B=-(\dot{R} / R) \cdot\left[(\ell+1) \dot{\rho}_{1}+\ell \dot{\rho}_{2}\right] /[(\ell$ +1) $\left.\rho_{1}+\ell \rho_{2}\right]$, the effect of convergence is properly ameliorated.

${ }^{5}$ M. E. Glinsky, D. S. Bailey, R. A. London, P. A. Amendt, A. M. Rubenchik, and M. Strauss, Phys. Fluids 13, 20 (2001).

${ }^{6}$ S. W. Haan, Phys. Rev. A 39, 5812 (1989).

${ }^{7}$ J. D. Lindl, Inertial Confinement Fusion (Springer-Verlag, New York, 1998).

${ }^{8}$ V. N. Goncharov, P. McKenty, S. Skupsky, R. Betti, R. L. McCrory, and C. Cherfils-Clérouin, Phys. Plasmas 7, 5118 (2000); H. Lin, B. C. Storey, and A. J. Szeri, Phys. Fluids 14, 2925 (2002).

${ }^{9}$ With $v_{r} \propto 1 / r^{2}$ and an assumed uniform density in the interior, the mass flux from a spherical volume surrounding the origin varies as $1 / r^{2}$. Thus, the rate of mass entering or leaving an infinitesimally small volume at the origin is nonzero, leading to a source or sink of material at the origin according to this model.
${ }^{10}$ P. Amendt, A. I. Shestakov, O. L. Landen, D. K. Bradley, S. M. Pollaine, L. J. Suter, and R. E. Turner, Phys. Plasmas 8, 2908 (2001).

${ }^{11}$ P. Amendt, J. D. Colvin, R. E. Tipton, D. E. Hinkel, M. J. Edwards, O. L. Landen, J. D. Ramshaw, L. J. Suter, W. S. Varnum, and R. G. Watt, Phys. Plasmas 9, 2221 (2002).

${ }^{12}$ R. E. Duff, F. H. Harlow, and C. W. Hirt, Phys. Fluids 5, 417 (1962).

${ }^{13}$ J. D. Ramshaw, Phys. Rev. E 61, 1486 (2000).

${ }^{14}$ F. Hattori, H. Takabe, and K. Mima, Phys. Fluids 29, 1719 (1986).

${ }^{15}$ D. Ofer, U. Alon, D. Shvarts, R. L. McCrory, and C. P. Verdon, Phys. Plasmas 3, 3073 (1996).

${ }^{16}$ O. L. Landen, C. J. Keane, B. A. Hammel, W. K. Levedahl, P. A. Amendt, J. D. Colvin, M. D. Cable, R. Cook, T. R. Dittrich, S. W. Haan, S. P. Hatchett, R. G. Hay, R. A. Lerche, R. McEachern, T. J. Murphy, M. B. Nelson, L. J. Suter, and R. J. Wallace, Phys. Plasmas 3, 2094 (1996); R. E. Chrien, N. M. Hoffman, J. D. Colvin, C. J. Keane, O. L. Landen, and B. A. Hammel, ibid. 5, 768 (1998).

${ }^{17}$ P. Amendt, R. E. Turner, and O. L. Landen, Phys. Rev. Lett. 89, 165001 (2002).

${ }^{18}$ J. D. Ramshaw, Phys. Rev. E 58, 5834 (1998); 61, 5339 (2000).

${ }^{19}$ R. E. Tipton, D. J. Steinberg, and Y. Tomita, Jpn. Soc. Mech. Eng. Int. J. Ser. II 35, 67 (1992).

${ }^{20}$ J. G. Clerouin, M. H. Cherfi, and G. Zerah, Europhys. Lett. 42, 37 (1998).

${ }^{21}$ H. F. Robey, Y. Zhou, A. C. Buckingham, P. Keiter, B. A. Remington, and R. P. Drake, Phys. Plasmas 10, 614 (2003).

${ }^{22}$ C. Paquette, C. Pelletier, G. Fontaine, and G. Michaud, Astrophys. J., Suppl. Ser. 61, 177 (1986).

${ }^{23}$ M. Brouillette and B. Sturtevant, J. Fluid Mech. 263, 271 (1994).

${ }^{24}$ R. D. Richtmyer, Commun. Pure Appl. Math. 8, 297 (1960).

${ }^{25}$ P. Carlès and S. Popinet, Phys. Fluids 13, 1833 (2001).

${ }^{26}$ K. O. Mikaelian, Phys. Rev. E 47, 375 (1993). 